\title{
PROJETO E ESTUDO DE CASO DA IMPLEMENTAÇÃO DE UM SISTEMA DE CONTROLE NEBULOSO
}

\begin{abstract}
Leandro dos Santos Coelho*
lscoelho@rla01.pucpr.br

ABSTRACT

This paper presents the design of a fuzzy control system applied to a multivariable nonlinear process and the state of art of fuzzy control applications in the industrial environment. The paper is divided in two parts. Initially the fuzzy control design is assessed on a horizontal balance process, a prototype in laboratory scale, with two propellers driven by two $D C$ motors. An overview of design aspects and characteristics of industrial applications of fuzzy controllers is also included.
\end{abstract}

\author{
Otacílio da M. Almeida ${ }^{\dagger}$ \\ otacilio@das.ufsc.br
}

\begin{abstract}
Antonio Augusto R. Coelho $\ddagger$
aarc@das.ufsc.br

*Laboratório de Automação e Sistemas - Programa de Pós-Graduação em Engenharia de Produção e Sistemas,

Pontifícia Universidade Católica do Paraná, Rua Imaculada Conceição, 1155 Prado Velho, 80215-901 - Curitiba, PR, Brasil

${ }^{\dagger}$ Departamento de Engenharia Elétrica - Universidade Federal do Ceará, Fortaleza, CE, Brasil

${ }_{\ddagger}^{\ddagger}$ Departamento de Automação e Sistemas - Universidade Federal de Santa Catarina, Caixa Postal 476, 88040-900 - Florianópolis, SC, Brasil
\end{abstract}

KEYWORDS: Fuzzy control, multivariable process, industrial applications.

\section{RESUMO}

Este artigo apresenta o projeto de um sistema de controle nebuloso aplicado a um processo não-linear multivariável e o panorama do estado da arte da aplicação de sistemas de controle na indústria. $\mathrm{O}$ artigo está dividido

\footnotetext{
Artigo submetido em 20/12/00

1a. Revisão em 15/01/01; 2a. revisão em 01/03/02

Aceito sob recomendação do Ed. Assoc. Prof. Fernando Gomide
}

em duas partes. Inicialmente é apresentado o projeto de um sistema de controle nebuloso aplicado ao processo balanço horizontal, um protótipo em escala de laboratório com dois ventiladores acionados por dois motores $D C$. A seguir, uma visão geral de aspectos de projeto e características de aplicações industriais de controladores nebulosos são apresentadas.

PALAVRAS-CHAVE: Controle nebuloso, processo multivariável, aplicações industriais.

\section{INTRODUÇÃO}

$\mathrm{Na}$ área de controle de processos o advento dos computadores propiciou a implementação de algoritmos que não poderiam ser configurados e aplicados utilizando-se a tecnologia analógica. As filosofias de controle feedforward, controle multivariável, controle ótimo, controle adaptativo e controle inteligente tornaram-se alternativas praticáveis. A proliferação das metodologias de controle denominadas de avançadas pode ser atribuída aos avanços realizados na indústria eletrônica, especialmente no desenvolvimento de dispositivos computacionais de baixo custo (anos 70). 
O que os usuários denominam de controle clássico e avançado são estratégias de controle dependente do marco de referência adotado. $\mathrm{Na}$ década de 40, consideravam-se controladores avançados os controladores que hoje em dia são classificados como controladores convencionais ou clássicos, entre os quais, controle em cascata, feedforward e compensadores avanço-atraso. Durante a década de 60, controle avançado significava algum algoritmo ou estratégia que difere do controlador PID (proporcional, integral e derivativo) clássico.

O projeto de algoritmos avançados, sob outro ponto de vista, descreve usualmente a combinação de elementos de muitas disciplinas, tais como engenharia de controle, processamento de sinais, estatística, teoria de decisão, inteligência computacional, entre outras. Muitos especialistas em controle denominam controladores avançados quando se referem aos controladores adaptativos, não-lineares, preditivos e inteligentes (Tovar, 1996). A Tabela 1 apresenta a classificação de controladores convencionais e avançados, segundo Unbehauen (1996), baseado na aceitação de controladores na indústria, predominantemente vinculado à indústria química.

Tabela 1: Classificação de controladores convencionais e avançados.

\begin{tabular}{|l|l|}
\hline projeto & tipo de controlador \\
\hline convencional & $\begin{array}{l}\text { manual, PID, proporcional, em } \\
\text { cascata e feedforward }\end{array}$ \\
\hline $\begin{array}{l}\text { avançado: clás- } \\
\text { sico }\end{array}$ & $\begin{array}{l}\text { escalonamento de ganhos, com- } \\
\text { pensação do atraso de trans- } \\
\text { porte, controle desacoplado }\end{array}$ \\
\hline $\begin{array}{l}\text { avançado: com } \\
\text { aplicações }\end{array}$ & $\begin{array}{l}\text { preditivo, modelo interno, adap- } \\
\text { tativo, nebuloso }\end{array}$ \\
\hline $\begin{array}{l}\text { avançado: poucas } \\
\text { aplicações }\end{array}$ & $\begin{array}{l}\text { não-linear, ótimo, robusto, inte- } \\
\text { ligente (neural e evolutivo) }\end{array}$ \\
\hline
\end{tabular}

As malhas de controle convencional têm sido utilizadas na maioria dos processos automatizados no meio industrial nos últimos 50 anos. Entre os motivos pode-se mencionar: estrutura simples, robustez, reduzido número de parâmetros a serem configurados, conhecimento intuitivo sobre o desempenho destas técnicas de controle, e requerimento mínimo de conhecimento matemático e técnico.

Entretanto, na teoria de controle convencional, os objetivos de projeto dos controladores são fixos e definidos pelo projetista. Consequentemente, a abordagem convencional de controle é limitada para muitas situações complexas, devido ao fato que estas requerem algumas formas de autonomia e as estratégias convencionais de controle oferecem um número reduzido de graus de li- berdade. Estas complexidades caracterizam processos com modelos matemáticos "pobres", elevados níveis de ruído, rigorosos índices de desempenho, presença de nãolinearidades, sensores distribuídos e a necessidade da tomada de decisões.

As metodologias avançadas permitem o aprimoramento de sistemas de controle comparado as técnicas convencionais, mas usualmente o projetista necessita ajustar uma quantidade de parâmetros de projeto que, em alguns casos, pode ser uma tarefa de difícil compreensão para não-especialistas na utilização destas técnicas de controle (Navarro e Albertos, 1996).

O aumento do número de aplicações de controle avançado tem sido motivado por diversos fatores vinculados a benefícios econômicos, tais como: projeto integrado de plantas e em sistemas de controle, flexibilidade na produção, aprimoramento do controle de qualidade, restrições ambientais, requerimentos de economia de energia, tentativas de maximização dos meios do setor produtivo (De Keyser, 1998). A análise destes fatores visa que os processos industriais sejam operados de maneira apropriada para atender a requisitos e normas de qualidade impostas por um mercado cada vez mais competitivo.

A partir dos anos 80 nota-se um acentuado crescimento do número de aplicações e sofisticação dos sistemas de controle avançados que empregam conceitos da teoria dos conjuntos nebulosos (fuzzy), denominados Sistemas Nebulosos $(S N s)$. A contribuição central dos $S N s$ é por caracterizar-se de uma metodologia apta ao tratamento de informação imprecisa, raciocínio aproximado, sistemas baseados em regras e manipulação de termos lingüísticos. Os $S N s$ são uma ferramenta poderosa possibilitando a expressão de conceitos que não sejam bem definidos, onde usualmente é empregado um nome ou adjetivo para descrevê-lo. A concepção lingüística da teoria dos $S N s$ habilita a fusão de processamento simbólico e computação numérica, provendo uma metodologia apta para problemas de decisão e controle, motivando a implementação de controladores nebulosos $(C N s)$.

Entre as vantagens dos $C N s$ sobre os controladores convencionais ou clássicos têm-se: (i) podem trabalhar com entradas contendo informações imprecisas; (ii) tratam processos complexos, com características de comportamento não-linear, elevada ordem, atraso de transporte, e sistemas "mal" definidos; e (iii) possibilitam a implementação do conhecimento e experiência de especialistas utilizando regras lingüísticas.

$\mathrm{O}$ artigo está dividido em duas partes. Na primeira parte, trata-se o estudo de caso experimental de $C N$ multivariável. O $C N$ é aplicado a um processo não- 
linear MISO (Multi-Input and Single-Output), denominado balanço horizontal, com características complexas do ponto de vista da engenharia de controle. Na segunda parte do artigo é apresentada uma visão geral das propostas da academia e um conjunto de aplicações industriais dos $C N \mathrm{~s}$.

\section{FUNDAMENTOS DE CONTROLE NE- BULOSO}

A teoria de controle clássica é adequada na resolução de problemas quando o processo é definido adequadamente e falha no tratamento de alguns processos complexos devido as não-linearidades e comportamentos variantes no tempo. Entretanto, operadores humanos freqüentemente controlam com sucesso processos complexos. Este fato motiva o projeto de controladores baseado na experiência de um operador humano.

A essência do controle nebuloso é explorar o conhecimento do operador humano (do processo) de forma a permitir a configuração de projetos de controle com desempenho satisfatório. $C N$ resulta no projeto de sistemas de controle não-lineares. Assim é uma tarefa difícil examinar a influência de cada parâmetro no desempenho e robustez do projeto. O procedimento de projeto adotado para um $C N$ baseado em regras é sintetizado pelos seguintes passos: (i) selecionar as entradas e saídas de controle; (ii) definir as funções de pertinência das entradas e das saídas; (iii) especificar as regras de controle; (iv) selecionar o método de inferência associado as regras de controle; (v) selecionar o método de nebulização e desnebulização; e (vi) avaliar o controlador.

O projeto de $C N s$ ou caracteriza-se pelo tratamento do conhecimento a priori sobre o processo (caso haja) ou é sintetizado através de algoritmos de otimização. Existem diversos métodos para o projeto de um $C N$, tais como: modelagem do conhecimento do engenheiro de controle, modelagem das ações de controle e experiência dos operadores humanos, e identificação do processo a ser controlado.

O resultado de um $C N$ baseado em regras apresenta uma superfície de controle codificada em uma base de conhecimento, sob forma de um conjunto de regras, termos lingüísticos e fatores de escala, sendo estas executadas por um controlador dedicado, interpretador ou compilador. O projeto dos $C N s$ apresenta um fluxo de dados (Figura 1) que passa pelas fases de: (i) nebulização (fuzzification); (ii) análise e execução de regras; e (iii) desnebulização (defuzzification).

A base de conhecimento abrange a base de regras e a

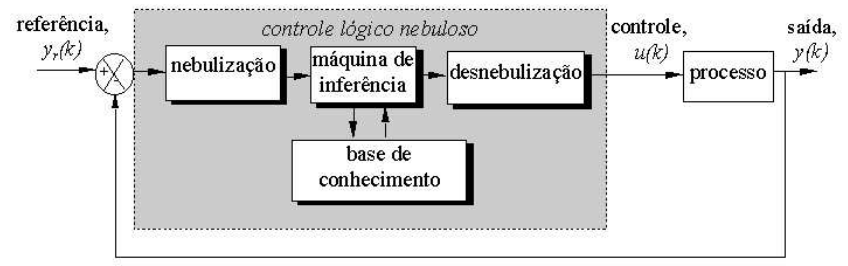

Figura 1: Configuração básica de $C N$ (Mamdani).

base de dados do $C N$. A base de regras é projetada pela seleção das variáveis de entrada, variáveis de saída e regras de controle. A base de dados é dependente da definição do universo de discurso de cada variável de estado, que está relacionada à seleção das funções de pertinência e dos parâmetros que as regem.

A fase de nebulização converte as variáveis numéricas em variáveis lingüísticas, ou seja, transforma os dados crisp em um conjunto nebuloso correspondente. As fases de execução de regras são responsáveis pela avaliação das regras de controle do tipo se <condição $>$ então $<$ conclusão $>$. Quando uma regra é ativada efetua-se um procedimento de cálculo baseado nos valores dos antecedentes e, então, é obtida a saída da regra. A fase de desnebulização - reverso da fase de nebulização transforma valores da saída do $C N$ em valores crisp.

\subsection{Exemplo do projeto de um CN}

\subsubsection{Descrição do processo multivariável}

O processo balanço horizontal $(P B H)$, ideal para aplicação em tempo real, é composto por uma haste metálica em cujas extremidades são montados dois motores $D C$ de 12 volts (controle $u_{1}$ e $u_{2}$ ) com hélices plásticas acopladas. O ponto central desta haste está preso ao eixo de um potenciômetro de precisão, acoplado a parte superior de outra haste metálica, vertical, fixa à base de apoio do protótipo. Esta base de apoio é formada por uma plataforma metálica, sobre a qual também está preso um estojo plástico que protege os circuitos de medição e atuação do processo, servindo de interface entre o controlador a ser utilizado, o usuário e o protótipo (Figura 2).

O potenciômetro de precisão é responsável pela medição do ângulo da haste. Com o movimento da haste no plano perpendicular ao potenciômetro, este fornece variações de resistência que são transformadas, por um circuito transmissor, em valores de tensão também padronizados na faixa de 0 a 5 volts, adequados ao controle digital. As saídas do controlador, também padronizadas em valores de 0 a 5 volts, são aplicadas aos motores, por intermédio 


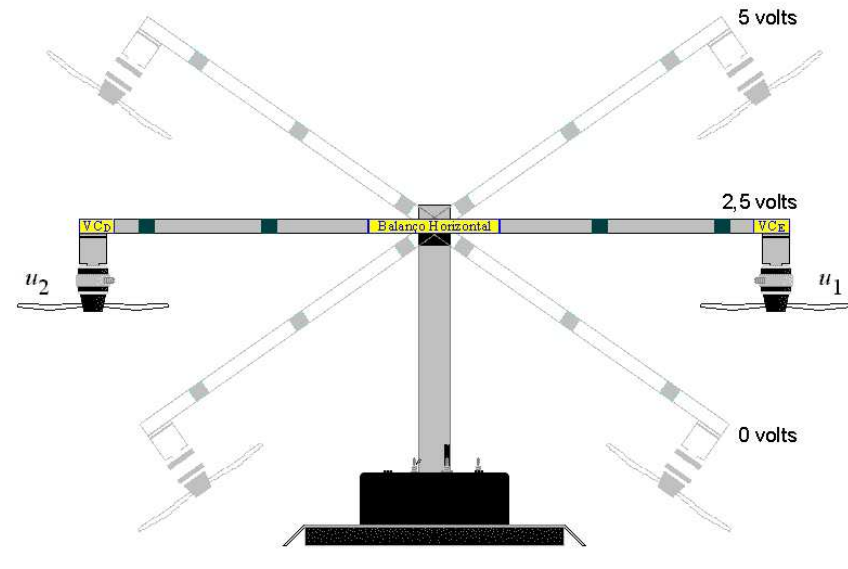

Figura 2: Visão do protótipo $P B H$.

de circuitos de acionamento, que transferem torque sobre o posicionamento da haste. Uma mola e uma massa de aproximadamente 30 gramas são inseridas no lado direito da haste do $P B H$ visando proporcionar diferentes pesos para cada lado da haste.

Este tipo de processo não-linear possui desafios para controladores que podem ser resumidos como segue: duplo integrador e instabilidade, não-linearidades, e a presença de perturbação no momento de inércia. Somente a ação de controle proporcional não assegura estabilidade sendo necessário a estrutura PID para garantir estabilidade e eliminação do erro em regime (Filatov et al., 1996). Estes problemas motivam a implementação de $C N s$. O desempenho e os experimentos realizados com o $C N$ abrangem apenas o comportamento servo (mudanças de referência).

\subsubsection{Projeto do controlador para o controle de um processo multivariável}

O projeto de um $C N$ para uma ou duas dimensões está bem consolidado por diversos trabalhos apresentados na literatura (Yager e Filev, 1994; Navarro e Albertos, 1996; Isermann, 1998; Hu et al., 1999), mas existem problemas quanto ao projeto de $C N s$ em aplicações multivariáveis, principalmente devido a: (i) limite da intuição do especialista para dimensões elevadas; (ii) complicações no tratamento matemático dos conceitos dos $S N s$ em problemas multivariáveis; (iii) crescimento do número de regras; e (iv) elucidação simplificada da base de regras para um operador é uma tarefa complexa (Gomide et al., 1995; Coelho 2000).

Recentemente, a literatura tem apresentado alguns estudos sobre a utilização de $S N s$ em controle de processos multivariáveis. Sabe-se que uma das maiores dificulda- des no projeto de $C N s$ multivariáveis está relacionado à dimensão e tamanho da base de regras, que muitas vezes pode se tornar impraticável para aplicações em tempo real. Procurando superar esta dificuldade, Linkens e Nyongesa (1996) propuseram um esquema hierárquico para o $C N$ multivariável. Utilizando este esquema, o $C N$ multivariável tem suas variáveis de estado (entradas) decompostas em pares, formando níveis intermediários de inferência que por sua vez são premissas para o próximo nível de inferência até a saída ser obtida. Pode-se mostrar que, o número de regras para o $C N$ multivariável hierárquico é uma função linear do número de entradas enquanto que, para o mesmo $C N$ multivariável implementado de forma convencional, a função é exponencial crescente (Linkens e Nyongesa, 1996). No mesmo enfoque, procurando superar o número excessivo de regras e sistematizar o projeto do controlador, Nie (1997) projetou um $C N$ multivariável utilizando a idéia de desacoplamento entre as malhas de controle e do controle descentralizado. Nesta abordagem, um $C N$ deve ser projetado para cada malha de controle que também utiliza desacopladores nebulosos. O $C N$ multivariável proposto por Qin et al. (1998) baseia-se no conceito de controle de modos deslizantes (sliding-mode control ? SMC) e na semelhança entre este e o conceito de controle nebuloso. Neste caso, o conjunto de regras do $C N$ são estabelecidas para controlar a distância entre os estados do sistema e o hiperplano de deslizamento (sliding hyperplane). O $C N$ multivariável proposto, assim projetado, foi utilizado para controlar um processo de forno de siderurgia.

O projeto de controle apresentado neste artigo é o $C N$ $(P D+I)$ incremental estendido para o caso MISO (Kwok et al., 1990; Malki et al.,1997). O $C N-(P D+I)$ é regido pela seguinte lei de controle:

$$
\begin{aligned}
u_{P I D}(k) & =u_{P D}(k)+u_{I}(k) \\
u_{P D}(k) & =-u_{P D}(k-1)+K u_{P D} \Delta u_{P D}(k) \\
u_{I}(k) & =u_{I}(k-1)+K u_{I} \Delta u_{I}(k)
\end{aligned}
$$

onde $u_{P I D}(k)$ é o vetor $(m \times 1)$ que representa a ação de $C N-P D$ nebulosa $+(I$ nebulosa $)$, constituída pela ação proporcional mais derivativa do erro, $u_{P D}(k)$, e a ação integral do erro $u_{I}(k)$. Os termos $K u_{P D}$ e $K u_{I}$ são as matrizes dos ganhos dos algoritmos de controle incremental proporcional mais derivativo e integral, respectivamente. O diagrama básico do $C N-(P D+I)$ incremental é apresentado na Figura 3. Embora a estrutura $C N-(P D+I)$ tenha sido utilizada, outras estruturas são possíveis: $C N-(P I+D), C N-(P I D), C N-P I, C N-(P D+I$ convencional). Entretanto, deve-se evitar a utilização do $C N-(P I D)$, devido à elevada quantidade de regras resultante da natureza tridimensional na base de regras 


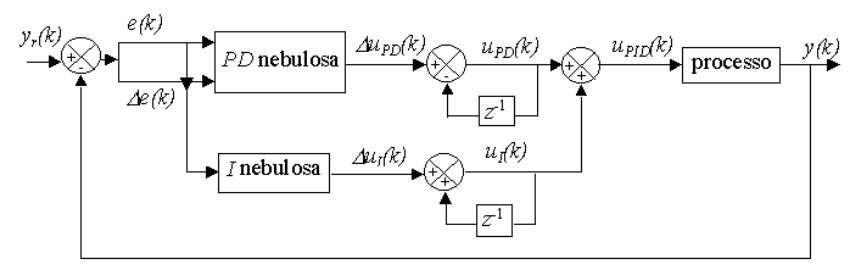

Figura 3: Diagrama do $C N-(P D+I)$ incremental.

(Almeida et al., 2000). A metodologia de projeto do $C N$ $(P D-I)$ incremental é descrita a seguir. Quatro aspectos relevantes são resolvidos para a concepção do projeto do controlador.

(1) Escolha das entradas e saídas: As características funcionais (i) e operacionais (ii) do controlador são determinadas e avaliadas, isto é,

(i) A seleção das variáveis de entrada e saída depende do controle a ser realizado. Neste caso tem-se o valor da inclinação da haste do $P B H$ como variável de saída (valores entre 0 e 5 volts). As entradas para o sistema de controle nebuloso são as variáveis erro e variação do erro da saída do processo em relação ao sinal de referência desejado. As duas saídas do controlador a serem aplicados ao $P B H$ (Figura 2) são os sinais de controle $u_{2}$ (aplicado ao motor do lado esquerdo) e $u_{1}$ (aplicado ao motor do lado direito).

(ii) O domínio dos valores das variáveis depende tanto do intervalo de valores normalizados das entradas do controlador como das ações de controle a serem aplicados ao processo. Neste estudo de caso, são introduzidos fatores de escala ajustáveis que são soluções pertinentes à seleção de valores adequados para este estudo prático. Os valores ajustados são: $K_{e}=4.5, K_{\Delta e}=0.1$, $K_{u P D 1}=K_{u P D 2}=5$ e $K_{u I 1}=K_{u I 2}=0.8$, com os mesmos valores para os dois controladores $u_{2}$ e $u_{1}$. Estes valores são multiplicados pelas funções de pertinência apresentadas na Figura 4.

(2) Definição das superfícies de controle: O número de conjuntos nebulosos foram escolhidos heuristicamente para reduzir a complexidade do controlador. Foram selecionados somente três conjuntos nebulosos para as entradas e saídas de forma a obter-se um desempenho satisfatório para o $C N$.

Para as variáveis lingüísticas são atribuídas os valores $P O$ sitivo $(P O)$, NEgativo $(N E), Z E$ ro $(Z E)$. As variáveis erro e $\Delta$ erro correspondem ao erro (diferença entre a referência e a saída do processo) e a variação do erro, respectivamente. O ajuste das funções de pertinência foi realizado por tentativa e erro. As configurações obti-

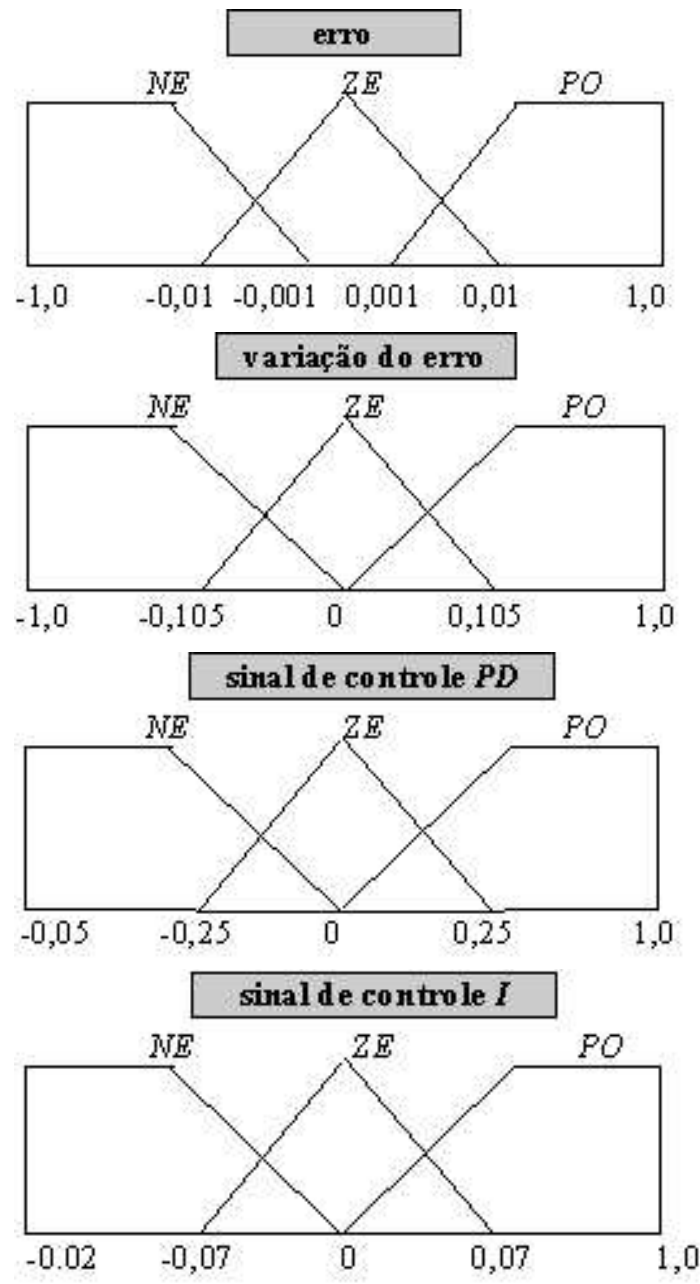

Figura 4: Funções de pertinência do $C N-(P D+I)$.

das para as funções de pertinência são apresentadas na Figura 4.

(3) Ajuste da base de regras: A estrutura multivariável

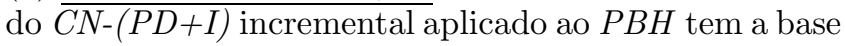
de regras apresentada na Figura 5 . A base de regras adotada para o controle $u_{1}$ é igual ao do controle $u_{2}$. As regras de produção utilizadas têm a forma $s e<c o n d i$ ção> então <conclusão $>$, por exemplo, se <erro é $P O$ e $\Delta$ erro é $Z E>$ então $<u_{1}$ é PO e $u_{2}$ é $N E>$.

(4) Escolha do método de nebulização e desnebulização: O método de desnebulização implementado é o centro da área (centróide) e a nebulização é realizada pela consulta a uma tabela previamente armazenada em memória.

Os resultados experimentais foram obtidos com um período de amostragem de $200 \mathrm{mseg}$. O ajuste dos fatores de escala, da base de regras e das funções de pertinência 


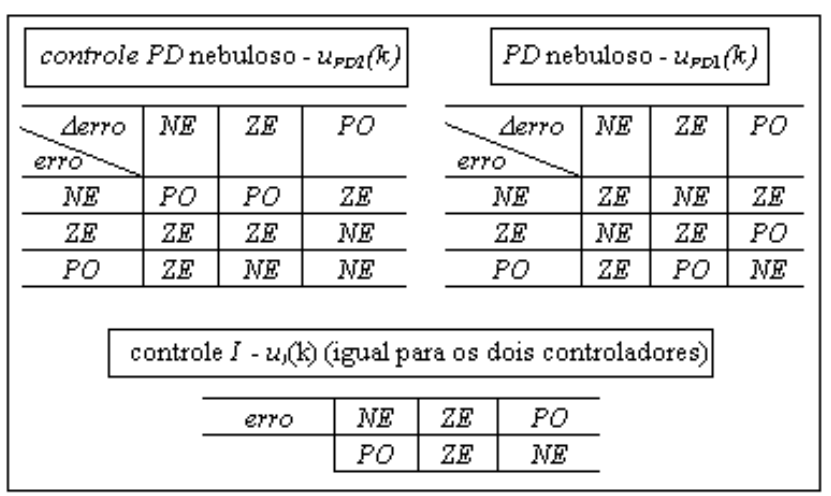

Figura 5: Base de regras do $C N-(P D+I)$.

de cada controlador foram realizados por ajustes finos e correções heurísticas vinculadas ao conhecimento sobre o processo a ser controlado. A base de regras do controlador $I$, no $C N-(P D+I)$, é constituído de 3 regras.

No ensaio submeteu-se o $P B H$ a variações de referências (comportamento servo). Inicialmente a referência foi estipulada em 4 volts, depois modificada para 1 volt, 3 volts, e finalmente 2 volts. A Figura 6 ilustra a saída, referência e a ação de controle para os experimentos de variação de referência do $P B H$ via $C N-(P D+I)$.

Nota-se pelo resultado apresentado que o controlador proporciona comportamento servo adequado no seguimento das referências. Entretanto, diversos fatores influenciam o desempenho do $C N$ apresentado, vinculados principalmente a seleção da base de regras, ajustes das funções de pertinência, determinação dos fatores de escala, divisão do universo de discurso e conhecimento a priori das características do processo pelo projetista (Coelho e Coelho, 1999).

\section{ASPECTOS IMPORTANTES DOS CONTROLADORES NEBULOSOS}

Em geral, o $C N$ é um tipo de estrutura de controle nãolinear, e deste modo possui o potencial de configurar um sistema de controle com desempenho equivalente ou superior as estratégias de controle linear. A seguir são apresentadas as características, os aspectos relevantes quanto as estruturas de $C N s$ e um conjunto das aplicações de $C N s$ no meio industrial.

\subsection{Classes de CNs}

Os classes de $C N s$ existentes são: Mamdani (Mamdani e Assilian, 1975) e Takagi-Sugeno (Takagi e Sugeno, 1985). Sob o enfoque estrutural estes $C N s$ dife-
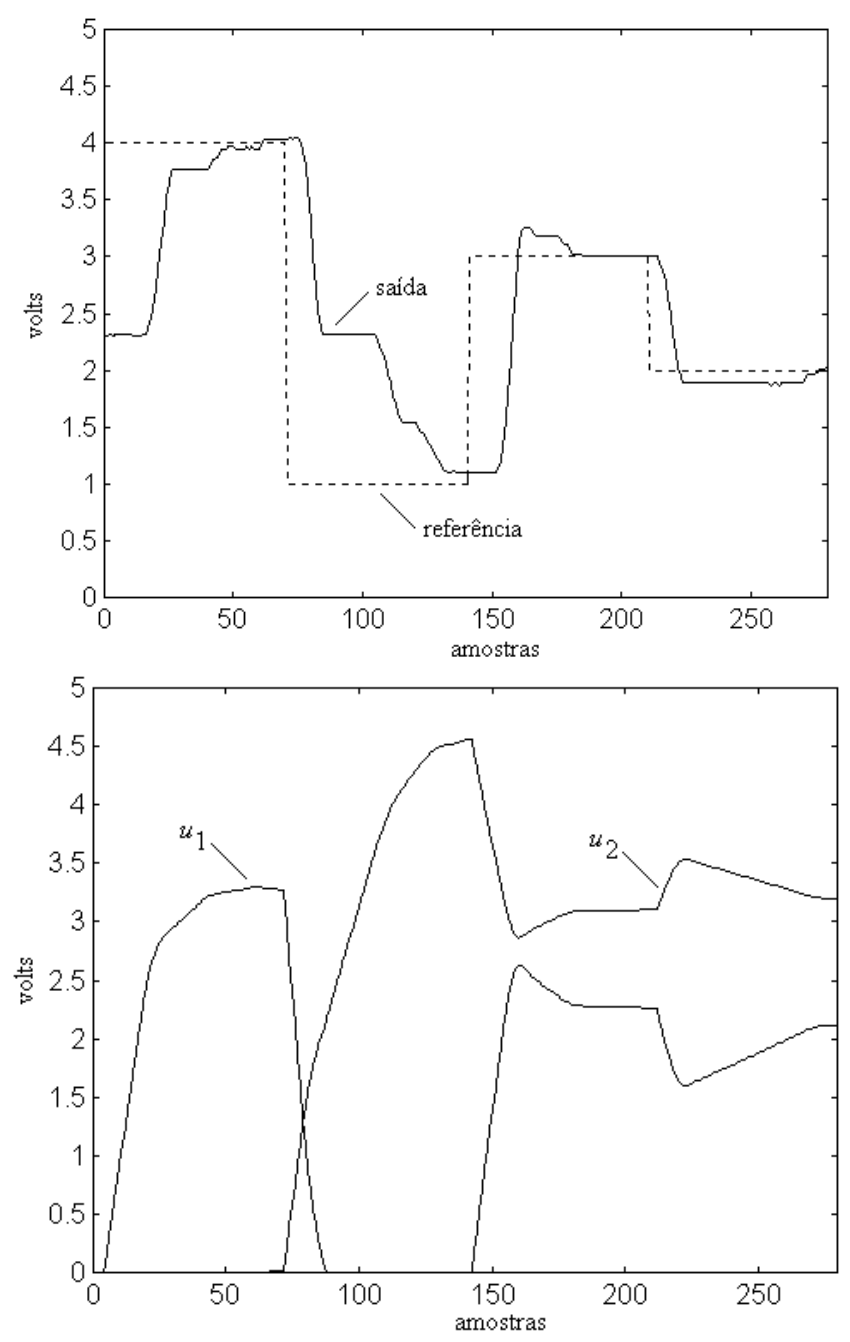

Figura 6: Ensaios para mudanças de referência do $\mathrm{PBH}$ via $C N-(P D+I)$.

rem principalmente quanto ao conseqüente das regras de controle. Os $C N s$ de Mamdani utilizam conjuntos nebulosos como conseqüentes, enquanto os controladores de Takagi-Sugeno empregam funções lineares como conseqüentes. Devido a esta diferença, as regras de controle de Mamdani são significativamente mais intuitivas linguisticamente, enquanto as regras de Takagi-Sugeno parecem apresentar mais poder de interpolação com um número reduzido de regras de controle. Ambos tipos de $C N s$ têm sido aplicados eficientemente na resolução de problemas práticos de controle (Ying, 1998).

\subsection{CN baseado em modelos nebulosos}

Existem dois tipos de conhecimento que podem ser utilizados para resolução de problemas complexos, conhe- 
cimento objetivo (representado por modelos matemáticos) e subjetivo (representado por informação lingüística que é usualmente complexa de quantificar pela matemática convencional). Estas duas formas de conhecimento podem ser coordenadas de maneira objetiva através da utilização dos $S N s$. As formas de abordar o conhecimento via $S N s$ são: abordagem baseada em modelo e abordagem livre de modelo.

A abordagem baseada em modelo nebuloso é obtida através de métodos de identificação de sistemas que levam em conta as medidas de entrada e saída do processo. Esta abordagem oferece uma alternativa aos modelos convencionais no tratamento de processos complexos. Os modelos nebulosos? modelos relacionais nebulosos, modelos lingüísticos ? podem ser utilizados para projeto de um controlador ou podem ser parte de uma estrutura de controle baseada em modelo. Exemplos são: (i) formas de controle local, baseados em mecanismos de escalonamento de ganhos; (ii) sistemas de controle para processos de fase mínima baseados em regras do tipo Mamdani podem ser projetados pela inversão de um modelo nebuloso do processo; e (iii) configuração de algoritmos de controle preditivo pela utilização de modelo nebuloso na forma de um preditor, sendo que as ações de controle são calculadas através de um algoritmo de otimização (Kiriakidis, 1998).

\subsection{Porquê utilizar controle nebuloso?}

As principais potencialidades que tornam os $C N s$ relevantes, podem ser enumeradas (Coelho e Coelho, 1999): (i) habilidade de controlar processos não-lineares: característica relevante à aplicação em identificação e controle de sistemas dinâmicos complexos, com características não-lineares e alta ordem; (ii) utilização de termos e expressões utilizados na linguagem natural; (iii) controle de processos quando o modelo matemático não é avaliado ou complexo à aplicação de metodologias da teoria de controle clássico; e (iv) possibilidade da implementação do conhecimento, aspectos intuitivos e experiência de especialista em controle utilizando-se regras lingüísticas (conhecimento qualitativo e estruturado) e entradas imprecisas (ao contrário das redes neurais onde o conhecimento é configurado de forma não-estruturada e quantitativa).

Contudo, os CNs apresentam algumas limitações: (i) alguns autores têm discutido que os $C N s$ são adequados onde o modelo matemático preciso do processo a ser controlado não é avaliado, mas é difícil projetar um controlador em que não necessita-se fazer considerações sobre seu ambiente; (ii) grande quantidade de parâmetros a ser configurado pelo usuário, tais como: número de subconjuntos de cada variável, número de regras, seleção do método para a realização das operações lógicas, método de desnebulização e parâmetros das funções de pertinência; e (iii) dificuldade da análise de aspectos de otimalidade, estabilidade e robustez dos $C N s$.

\subsection{CN é uma realidade no meio industrial}

Os fundamentos teóricos dos conjuntos nebulosos propostos por L. Zadeh em 1965, como uma forma alternativa de se modelar sistemas complexos e difíceis de serem descritos por técnicas convencionais, motivaram inicialmente a validação dos $C N$ s por Mamdani e Assilian (1975).

A primeira aplicação industrial de $C N$ foi o controle de um forno para produção de cimento pela Smidth Co. Ltda em Copenhague, Dinamarca (Holmblad e Ostergaad, 1982). A metodologia baseada em $C N$, neste caso, visou a aquisição da experiência operacional dos operadores e engenheiros de controle no controle manual do processo industrial. Após este trabalho pioneiro, as vantagens e simplicidade do projeto dos $C N$ s motivaram o emergente desenvolvimento acadêmico e comercial de ambientes e ferramentas de software e hardware com a utilização da tecnologia dos $S N s$. As mais variadas áreas do conhecimento, principalmente no Japão, (país pioneiro na utilização em larga escala destes produtos) e mais recentemente nos EUA e Europa, utilizam esta tecnologia (Bonissone, 1994; Gomide et al., 1995; Hirota e Sugeno, 1995; Bartos, 1997).

Atualmente, uma grande variedade de produtos industriais e comerciais, baseados em lógica nebulosa são disponíveis. Exemplos típicos incluem produtos de consumo direto tais como máquinas de lavar roupas (Sanyo), máquinas de lavar louças (Hitachi), geladeiras (Sharp), ar condicionados (Mistisubichi), fornos de microondas (Sanyo), câmaras de vídeo (Canon, Panasonic), aspiradores de pó (Matsushita), etc. Na indústria automotiva destaca-se, transmissão automática (Nissan, Lexus), injeção eletrônica, suspensão ativa, veículos auto-guiados e robôs móveis $(N A S A, I B M)$, etc. $\mathrm{Na}$ linha de automação predial pode-se citar o controle de grupos de elevadores (Hitachi, Toshiba), controle de motores (Hitachi), sistema de ventilação de túneis urbanos (Toshiba), controle de tráfego urbano e controle de partida e parada de trens urbanos e metrôs (Sendai, Tokio), etc.

Em termos de equipamentos manufaturados para controle e automação industrial, o primeiro controlador lógico programável ? PLC (Programable Logic Controller) ? a incorporar a lógica nebulosa foi o ES100 proje- 
tado pela OMRON. O $C N$ no ES100 apresenta-se combinado com um controlador PID de forma que o controlador nebuloso resultante é não-linear, auto-ajustável e apresenta desempenho superior ao controlador PID convencional linear, frente as não-linearidades inerentes ao processo controlado. O PLC ES100 é completamente programável, monitorado por software e todas as funções básicas são disponíveis a partir do painel frontal do equipamento. O ES100 vem com rotinas internas que implementam algoritmos do tipo rampa, permite a adição de módulos de expansão de entradas e saídas ao sistema e são equipados com portas de comunicação para conexão em rede industrial. O módulo básico do $P L C(C P U)$ vem equipado com termopares $(K, J$, $P, E, L, V, R, S, B, W, P L)$, termistores (PT100 e JPT100), conversores $A / D$ e $D / A$ (em corrente e em tensão) (Gebhardt e Altrock, 1996).

Outros fabricantes de $P L C$ 's já incorporaram capacidade de controle nebuloso em seus controladores industriais entre estes pode-se citar a série $C 1, M 1, M 2, M 3$, $M 4$ e $M 5$ da $A S C O N$ que são mini- $P L C$ 's, programados e monitorados por software em um ambiente windows. Adicionalmente, possuem entradas tipo termopares, termistores, $A / D$ e $D / A$ de propósito geral e saídas tipo relé contato seco, triacs e lógicas, além de portas de comunicações para configuração em redes industriais. Outro exemplo é o controlador fuzzyPLC da Kroner Moeller. Este $P L C$ é equipado com entradas e saídas digitais e analógicas configuráveis e são capazes de monitorar mais de 100 pontos de entradas e saídas através de módulos de expansões. Uma importante característica do fuzzyPLC corresponde a sua capacidade de ser programado e monitorado através do software fuzzyTECH que executa num ambiente windows e programa uma série de microcontroladores e controladores de processos.

\section{CONCLUSÃO}

Um esquema de $C N-(P D+I)$ incremental foi apresentado e aplicado a um processo experimental multivariável, caracterizado por instabilidade, fase não-mínima e presença de não-linearidades. O projeto do $C N-(P D+I)$ incremental apresentou comportamento adequado na tarefa de controle do $\mathrm{PBH}$.

Aspectos relativos as potencialidades, limitações e a realidade no meio industrial quanto a utilização de $C N s$ em ambientes industriais também foram discutidas. O $C N$ é uma realidade na indústria, mas a principal dificuldade da sua utilização reside na ausência de ferramentas padronizadas para o desenvolvimento de tais controladores (Navarro e Albertos, 1996). Entretanto existem perspectivas futuras em relação ao projeto de $C N s$ que englobam intensas pesquisas nos seguintes tópicos:

- verificação de aspectos relativos à estabillidade: As questões de estabilidade são um dos mais importantes aspectos na análise e projeto de sistemas de controle nebulosos. A análise de estabilidade de $C N s$ é complexa porque estes sistemas são essencialmente não-lineares. Contudo, recentemente, alguns resultados de estabilidade não-linear têm sido relatados, inclusive para o tratamento de processos multivariáveis. Por exemplo, para projetar um $C N$ multivariável estável, Cheng e Rees (1997) utilizaram modelos lineares para cada ponto de operação do processo (linearização por partes). Estes modelos são então conectados através de sistemas nebulosos para a formação de um modelo geral e, a partir daí, pode-se provar, através da teoria de funções de Lyapunov, que o $C N$ resulta um sistema globalmente estável. Fischle e Schröder (1999) apresentaram um esquema de projeto de $C N s$ multivariáveis adaptativos que resultam em garantias, em termos de funções de Lyapunov, de convergência da saída do sistema para um sinal de referência estabelecido. Cuesta et al. (1999) propuseram um método de projeto de $C N$ multivariável baseado no modelo de Takagi-Sugeno para o qual conclusões sobre a estabilidade podem ser estabelecidas através da formulação de Lur, critério do círculo para sistemas multivariáveis e balanço harmônico.

- algoritmos de otimização: O desenvolvimento de algoritmos de otimização eficientes é um tópico de intensas pesquisas, abrangendo métodos dos mínimos quadrados não-linear, algoritmos recursivos, métodos baseados em gradiente, algoritmos evolutivos, entre outros.

- projeto combinado com abordagens de controle preditivo e robusto: As principais dificuldades no projeto de controladores para sistemas complexos é o tratamento das não-linearidades e parâmetros variantes no tempo. Estes fatores motivam a abordagem robusta no projeto de $C N s$, com técnicas baseadas em controle adaptativo, preditivo (Roubos et al., 1998), teoria de controle $H^{\infty}$, utilização de algoritmos de programação convexa em abordagens via linear matrix inequalities e estabilização quadrática (Tanaka et al., 1996).

- controle hierárquico: A análise e projeto de $C N s$ hierárquicos para aplicações em controle de processos multivariáveis é uma abordagem relevante. Nesta abordagem pode-se diminuir a complexidade do projeto do $C N$ com um número menor de regras pela decomposição do controlador em uma estrutura em cascata. Além disso, segundo Wang (1999), pode-se provar que $S N$ hierárquicos são aproximadores universais.

- controle por modos deslizantes: O controle por modos 
deslizantes é um caso especial do controle de estrutura variável, em que as propriedades de diferentes estruturas de controle podem ser utilizadas à síntese de um sistema de controle estável. As conexões entre os $C N s$ e controle por modos deslizantes podem ser realizadas com as abordagens direta e indireta (Jiwei et al., 1999).

- hidridização com outras metodologias da inteligência computacional: O progresso da tecnologia dos sistemas inteligentes é motivado pela necessidade do desenvolvimento de estratégias flexíveis e eficientes com o propósito de tratar aplicações complexas. Cada metodologia da inteligência computacional possui potencialidades e limitações que as tornam mais adequadas para algumas aplicações particulares. Estas limitações são a principal motivação da criação de sistemas híbridos inteligentes, onde duas (ou mais) técnicas são combinadas para superar as limitações das técnicas quando tratadas individualmente. Os sistemas híbridos inteligentes são relevantes quando se considera a natureza variada de aplicações complexas. Entre as formas dos sistemas híbridos inteligentes que tem sido alvo de pesquisas emergentes incluem-se sistemas evolutivo-nebulosos, sistemas evolutivo-neurais, sistemas neuro-nebulosos e sistemas neuro-nebuloso-evolutivos.

\section{AGRADECIMENTOS}

Os autores agradecem as contribuições dos revisores para o aprimoramento do artigo.

\section{REFERÊNCIAS}

Almeida, O. M., L. S. Coelho e A. A. R. Coelho (2000). Some issues concerning design and evaluation of fuzzy controllers, Anais do IV Industry Applications Conference, Porto Alegre, RS, pp. 168-173.

Bartos, F. J. (1996). Fuzzy logic reaches adulthood, Control Engineering, July, pp. 50-56.

Bartos, F. J. (1997). Artificial intelligence: smart thinking for complex control, Control Engineering, July, pp. $44-52$.

Bonissone, P. P. (1994). Fuzzy logic controllers: an industrial reality, Computational Intelligence: Imitating Life, Zurada, J. M.; Marks II, R. J.; Robinson, C. J. (eds.), Piscataway, NJ, IEEE Press, pp. 316327 .

Coelho, L. S. (2000). Identificação e controle de processos multivariáveis via metodologias avançadas e inteligência computacional, Tese de doutorado,
Departamento de Automação e Sistemas, Universidade Federal de Santa Catarina.

Coelho, L. S. e A. A. R. Coelho (1999). Fuzzy PID controllers: structures, design principles and application for nonlinear practical process, Advances in soft computing - engineering design and manufacturing, Roy, R., Furuhashi, T. e P. K. Chawdhry (eds.), Springer-Verlag, London, pp. 147-159.

Cheng, C. M. e N. W. Rees (1997). Stability analysis of fuzzy multivariable systems: vector Lyapunov function approach, IEE Proceedings Control Theory and Applications, Vol. 144, No. 5, pp. 403-412.

Cuesta, F., F. Gordillo, J. Aracil e A. Ollero (1999). Stability analysis of nonlinear multivariable TakagiSugeno fuzzy control systems, IEEE Transactions on Fuzzy Systems, Vol. 7, No. 5, pp. 508-520.

De Keyser, R. (1998). A gentle introduction to model based predictive control, International Workshop on Constrained Control Systems, Neccosyde: Alfa Network for Constrained Control System Development, Florianópolis, SC.

Filatov, N. M., U. Keuchel e H. Unbehauen (1996). Dual control for an unstable mechanical plant, IEEE Control Systems, Vol. 16, No. 4, pp. 31-37.

Fischle, K. e D. Schröder (1999). An improved stable adaptive fuzzy control method, IEEE Transactions on Fuzzy Systems, Vol. 7, No. 1, pp. 27-40.

Gebhardt, J. e C. Altrock (1996). Fuzzy application library/technical applications/industrial automation, Proceedings of 5th IEEE International Conference on Fuzzy Systems, New Orleans.

Gomide, F. A. C. e R. R. Gudwin (1994). Modelagem, controle, sistemas e lógica fuzzy, Revista da $S B A$ Controle \&G Automação, Vol. 4, No. 3, pp. 97-115.

Gomide, F. A. C., R. R. Gudwin e R. Tanscheit (1995). Conceitos fundamentais da teoria de conjuntos fuzzy, lógica fuzzy e aplicações. Proceedings of 6th International Fuzzy Systems Association World Congress - IFSA95, Tutorials, pp. 1-38.

Hirota, K. e M. Sugeno (1995). Industrial applications of fuzzy technology in the world, advances in fuzzy systems? applications and theory, Singapore, World Scientific, Vol. 2.

Holmblad, L. P. e J. -J. Ostergaard (1982). Control of a cement kiln by fuzzy logic. Fuzzy information and decision processes, Gupta, M. M. e E. Sanchez (eds.), Amsterdam, North-Holland, pp. 389-399. 
Hu, B., G. K. I. Mann e R. G. Gosine (1999). New methodology for analytical and optimal design of fuzzy PID controllers, IEEE Transactions of Fuzzy Systems, Vol. 7, No. 5, pp. 521-539.

Isermann, R. (1998). On fuzzy logic applications for automatic control, supervision, and fault diagnosis, IEEE Transactions on Systems, Man, and Cybernetics? Part A: systems and humans, Vol. 28, No. 2, pp. 221-235.

Kiriakidis, K. (1998). Fuzzy model based control of complex plants, IEEE Transactions on Fuzzy Systems, Vol. 6, No. 4, pp. 517-529.

Kwok, D. P., P. Tam, C. K. Li e P. Wang (1990). Linguistic PID controllers, 11th IFAC World Congress, Tallinn, Estonia, USSR, Vol. 7, pp. 192-197.

Jiwei, W-., Lihong, X-. e X-. Yunshi (1999). A new design method of fuzzy sliding mode controller with faster convergence, Proceedings of IEEE International Fuzzy Systems Conference, Seoul, Korea, Vol. 1, pp. 96-99.

Linkens, D. A. e H. O. Nyongesa (1996). A hierarchical multivariable fuzzy controller for learning with genetic algorithms, International Journal of Control, Vol. 63, No. 5, pp. 865-883.

Malki, H. A., D. Misir, D. Feifenspan e G. Chen (1997). Fuzzy PID control of a flexible-joint robot arm with uncertainties from time-varying loads, IEEE Transactions on Control Systems Technology, Vol. 3, No. 3, pp. 371-378.

Mamdani, E. e S. Assilian (1975). An experiment in linguistic synthesis with a fuzzy logic controller, International Journal of Man Machine Studies, Vol. 7, No. 1, pp. 1-13.

Navarro, J. L. e P. Albertos (1996). Fuzzy logic implementation of industrial controllers, 13th IFAC World Congress, San Francisco, CA, pp. 409-414.

Nie, J. (1997). Fuzzy control of multivariable nonlinear servomechanisms with explicit decoupling scheme, IEEE Transactions on Fuzzy Systems, Vol. 5, No. 2, pp. 304-311.

Qin, Y., Jia, L. -M. e X. -D. Zhang (1998). Automation of combustion process for coke oven using multivariable fuzzy control technique, Proceedings of IEEE World Congress on Computational Intelligence, FUZZ-IEEE, Anchorage, Alaska, pp. 606610.
Roubos, J. A., R. Babuska, P. M. Brujin e H. B. Verbruggen (1998). Predictive control by local linearization of a Takagi-Sugeno fuzzy model, Proceedings of IEEE World Congress on Computational Intelligence, FUZZ-IEEE, Anchorage, Alaska, pp. 37-42.

Takagi, T. e M. Sugeno (1985). Fuzzy identification of systems and its applications to modeling and control, IEEE Transactions on System, Man and Cybernetics, Vol. 15, No.1, pp. 116-132.

Tanaka, K., T. Ikeda e H. O. Wang (1996). Robust stabilization of a class of uncertain nonlinear systems via fuzzy control: quadratic stability, $H^{\infty}$ control theory, and linear matrix inequalities, IEEE Transactions on Fuzzy Systems, Vol. 4, No. 1, pp. 1-13.

Tovar, S. N. (1996). El control avanzado en los procesos industriales, Boletin Informativo de la Asociacion Colombiana de Automatica, No. 3, pp. 2-3.

Unbenhauen (1996). Distributed supervisory industrial control systems and their problems for embedding advanced control strategies, $7^{\circ}$ Congreso Latinoamericano de Control Automatico e XV Simposio Nacional de Control Automatico, Buenos Aires, Argentina, Vol. 1, pp. I-VIII.

Yager, R. R. e D. P. Filev (1994). Essentials of fuzzy modeling and control, John Wiley \& Sons: New York, NY.

Ying, H. (1998). The Takagi-Sugeno fuzzy controllers using the simplified linear control rules are nonlinear variable gain controllers, Automatica, Vol. 34, No. 2, pp. 157-167.

Wang, L. -X. (1999). Analysis and design of hierarchical fuzzy systems. IEEE Transactions on Fuzzy Systems, Vol. 7, No. 5, pp. 617-624.

Zadeh, L. A. (1965). Fuzzy sets, Information and Control, Vol. 8, pp. 338-353.

Zadeh, L. A. (1994). Why the success of fuzzy logic is not paradoxical, IEEE Expert, Vol. 9, No. 4, pp. 43-46. 